\title{
How Many PET Tracers Do We Need?
}

\author{
Markus Schwaiger ${ }^{1}$ and Hans-Jürgen Wester ${ }^{2}$ \\ ${ }^{I}$ Nuklearmedizinische Klinik und Poliklinik, Klinikum Rechts der Isar, Technische Universität München, München, Germany; and \\ ${ }^{2}$ Lehrstuhl für Pharmazeutische Radiochemie, Technische Universität München, Garching, Germany
}

The recognized need for new PET tracers is associated with increases in available PET instrumentation and with unmet clinical challenges in the early diagnosis and staging of diseases. The clinical success of ${ }^{18} \mathrm{~F}$-FDG PET has resulted in the acceptance of biologic signals as part of disease management. The advantages of PET technology over SPECT will lead to the introduction of new PET tracers for established nuclear medicine imaging indications. Disease-specific markers such as amyloid ligands will lead to new applications of PET to neurodegenerative diseases that are prevalent in aging societies. The translation of molecular imaging to clinical applications will require combining specific tracer approaches with targeted therapy for the realization of personalized medicine. An important aspect of the introduction of new PET tracers will be the emergence of a specialized radiopharmaceutical industry and professional distribution networks. The number of available PET tracers not only will follow rules of demand and supply but also will be dependent on the regulatory environment of individual health care systems.

Key Words: PET; diagnostic single-photon imaging; therapeutic radiopharmaceuticals

J Nucl Med 2011; 52:36S-41S

DOI: 10.2967/jnumed.110.085738

\section{I}

maging has become an integral part of medicine because it is used for the detection, staging, and monitoring of diseases. Advances in imaging instrumentation have resulted in superb spatial and temporal resolution of image generation. Most imaging procedures necessitate the use of contrast agents to improve noninvasive tissue characterization. Nuclear medicine imaging procedures are based on the tracer principle, which allows the visualization of biologic and physiologic processes with small amounts of specific radiopharmaceuticals. Because the production and application of radiopharmaceuticals are regulated with principles similar to the general rules and guidance used for pharmaceuticals, the development and approval of new radiopharmaceuticals are associated with considerable costs for development and validation

Received Feb. 18, 2011; revision accepted May 26, 2011.

For correspondence or reprints contact: Markus Schwaiger, Nuklearmedizinische Klinik und Poliklinik, Klinikum Rechts der Isar, Technische Universität München, Ismaningerstrasse 22, 81675 München, Germany.

E-mail: markus.schwaiger@tum.de

COPYRIGHT @ 2011 by the Society of Nuclear Medicine, Inc. processes, including clinical evaluation. This regulatory process has been traditionally addressed by the radiopharmaceutical industry, which supplies the nuclear medicine community with approved and often patented radiopharmaceutical products. In Germany, there are currently 93 commercially available individual radiopharmaceutical products for diagnostic single-photon imaging and 23 therapeutic radiopharmaceutical products with approved indications.

Besides this clinical market for single-photon and therapeutic radiopharmaceuticals, the tracer principle has gained wide acceptance within the research community because of the high sensitivity of radioactivity detection and the biologic specificity of targeted tracers. Many radioactive compounds for laboratory use are commercially available and are used worldwide for in vitro and in vivo preclinical work. On the basis of the experimental success of ${ }^{14} \mathrm{C}$-labeled substances, such as ${ }^{14} \mathrm{C}$-deoxyglucose, for quantitative autoradiography of the brain, PET with ${ }^{11} \mathrm{C}$ - and ${ }^{18} \mathrm{~F}$-labeled compounds was introduced in the late 1970s as a tool for translating quantitative autographic imaging into a clinical imaging method $(1,2)$. PET was initially applied to clinical research in neuroscience and cardiology. Many cyclotrons were installed at academic institutions for the on-site production of short-lived PET radiopharmaceuticals (3), and many different ${ }^{11} \mathrm{C}$ and ${ }^{18} \mathrm{~F}$ tracers were designed for individual research projects and relatively limited clinical applications. With the recognition of the clinical potential of ${ }^{18} \mathrm{~F}-\mathrm{FDG}$ for the detection of malignant tissue through the Warburg effect, PET quickly became a standard diagnostic procedure in oncology. With this success came a need for the commercial distribution of ${ }^{18} \mathrm{~F}-\mathrm{FDG}$ as a radiopharmaceutical to supply users without cyclotrons. Because ${ }^{18} \mathrm{~F}-\mathrm{FDG}$ is a nonpatented tracer, local production through commercial and academic radiopharmacies with manufacturing licenses became the business model for the supply and distribution of ${ }^{18} \mathrm{~F}$-FDG.

With the success of ${ }^{18} \mathrm{~F}$-FDG PET in oncology and the rapid expansion of clinically available PET/CT instrumentation worldwide, clinical interest in new PET radiopharmaceuticals is continuously growing.

\section{HOW MANY PET TRACERS DO WE NEED?}

Here we do not discuss the need for PET tracers for use in individuals' research projects; rather, we address this topic with 3 hypotheses that define the future need for PET radiopharmaceuticals for clinical applications: 
1. PET technology is superior to single-photon imaging and will challenge SPECT in advanced health care markets supported by a specialized radiopharmaceutical industry and distribution networks.

2. The growth of PET will be accompanied by the increasing availability of PET tracers for established nuclear medicine imaging indications (e.g., bone scanning and myocardial perfusion imaging) and the development of new PET probes for the diagnosis and staging of prevalent diseases (e.g., amyloid ligands).

3. The success of translating molecular PET approaches to use in patients will depend significantly on the integration of therapeutic strategies and new imaging methodology and their impact on therapeutic decision making for the realization of personalized medicine.

PET offers distinct advantages over SPECT, and those advantages will increase the relative use of PET technology. Higher sensitivity of the instrumentation together with quantification of regional tissue tracer concentrations will maximize the use of the tracer principle to visualize and measure biologic processes. The integration of PET technology with CT and MRI not only improves diagnostic work flow and the coregistration of imaging data but also affects the accuracy of PET measurements by providing accurate attenuation maps and high-resolution anatomic information for partial-volume correction. The resulting quantitative information will produce more accurate criteria for diagnosis and assessment of the extent of disease and will allow therapy monitoring based on longitudinal measurements of biologic parameters. Short-lived isotopes and higher sensitivity of PET radioactivity detection will reduce radiation exposure in many instances and shorten acquisition time, providing further improvements in image quality and diagnostic work flow. The speed of the transition from SPECT to PET will depend on the cost of the instrumentation and on national reimbursement strategies allowing widespread clinical applications. Given reimbursement, the higher cost of instrumentation will be easily offset by the high throughput of patients that is possible with state-of-the-art PET/CT instrumentation, as has been demonstrated with ${ }^{18} \mathrm{~F}-\mathrm{FDG}$ PET.

One major hurdle for the further expansion of clinical applications for PET is the lack of innovative and approved radiopharmaceuticals beyond ${ }^{18} \mathrm{~F}-\mathrm{FDG}$. Because the production of ${ }^{18} \mathrm{~F}-\mathrm{FDG}$ is associated with little profitability, the financial investments necessary for the creation of a dedicated PET radiopharmaceutical industry depend on a business model with patented tracers and professional distribution networks. Therefore, the question "How many PET tracers do we need?" may be more adequately rephrased as the question "How many PET tracers can we afford?" The costs of establishing new radiopharmaceuticals have been raised not only by the worldwide tightening of good manufacturing practice regulations and the corresponding extended infrastructure requirements but also by the need for comprehensive manufacturing authorizations and the high costs of clinical validation and approval processes. Consequently, a consolidation of radiopharmaceutical production capacities likely will take place, resulting in a reduction in the number of nonindustrial academic producers. On the other hand, there are many existing local suppliers of PET radiopharmaceuticals, especially in Europe, with limited resources for the required investments in tracer development and clinical trials. The market for PET radiopharmaceuticals is relatively small in comparison with the drug market and is locally very heterogeneous because of national differences in reimbursement for PET. The process of consolidation and emergence of an international PET radiopharmaceutical industry is just beginning but may gain momentum with the pending approval of new radiopharmaceuticals, such as ${ }^{18} \mathrm{~F}$-labeled amyloid tracers and ${ }^{18} \mathrm{~F}$-labeled myocardial perfusion agents. In addition, there is the hope in academia that the strict interpretation of pharmaceutical laws and the corresponding guidelines may be eased for the production and use of short-lived PET radiopharmaceuticals in academic pharmacies and, thus, may reduce the infrastructure requirements for pharmaceutical production in research institutions. Addressing the regulatory and economic issues associated with the production of new radiopharmaceuticals requires a coordinated strategy that not only develops partnerships between academia and industry but also convinces public funding agencies, such as the National Institutes of Health and the European Commission, to support the clinical validation of new radiopharmaceuticals.

\section{F PET Tracers}

The physical characteristics of ${ }^{18} \mathrm{~F}$ have supported the centralized synthesis of ${ }^{18} \mathrm{~F}$-labeled compounds and the subsequent distribution of those compounds to PET operations. Numerous networks for the distribution of ${ }^{18} \mathrm{~F}$-labeled compounds already exist worldwide; therefore, ${ }^{18} \mathrm{~F}$-labeled compounds will continue to play an important role in the transition from SPECT to PET. Compared with state-ofthe-art bone-seeking SPECT agents, ${ }^{18} \mathrm{~F}$-sodium fluoride has been shown in many studies to provide more sensitive detection of bony abnormalities and may, in combination with PET/CT and MRI/PET instrumentation, be of special interest for replacing standard bone scintigraphy, which represents the "backbone" of many nuclear medicine services. Cost efficiency studies and clinical marketing will determine the future of this tracer because international approval and reimbursement are likely to follow the U.S. example of a national registry in response to the current molybdenum shortage (http://www.cancerpetregistry.org/).

Cardiology. ${ }^{18} \mathrm{~F}$-labeled radiopharmaceuticals are currently in clinical evaluations for myocardial perfusion imaging and are expected to be commercially available within the next few years. There is no question that there will be a role for PET in cardiology because of the success of myocardial perfusion imaging with SPECT and the clinical acceptance 
of ${ }^{82} \mathrm{Rb}$ PET in the United States. However, ${ }^{82} \mathrm{Rb}$ generators are relatively costly and require a dedicated PET operation to be cost-effective. The methodologic integration of quantitative PET myocardial perfusion measurements with CT angiography in the decision-making process for patients with suspected or proven coronary artery disease is currently being discussed and will be an attractive diagnostic option for cardiologists. We are convinced of the need for ${ }^{18} \mathrm{~F}-$ labeled myocardial perfusion tracers in nuclear cardiology. Fortunately, the first such tracer is in development and will be available as a patented product in the near future (4). If ${ }^{123}$ I-metaiodobenzylguanidine SPECT becomes an accepted imaging tool for patients who have heart failure and are at risk for sudden death, then the corresponding ${ }^{18} \mathrm{~F}$-labeled PET compound is needed and will follow. There is a documented need to develop imaging techniques to select patients for the implantation of cardiac defibrillators.

Neurology. The need for ${ }^{18} \mathrm{~F}$ substitutes also applies to the established ${ }^{99 \mathrm{~m}} \mathrm{Tc}$-labeled compounds used in neurology. For example, the identification of recurrent tumor growth in patients with glioblastoma is a well-appreciated clinical challenge for which improved diagnostic tools are needed. ${ }^{18} \mathrm{~F}-$ labeled amino acids such as ${ }^{18} \mathrm{~F}$-ethyltyrosine have been well characterized and shown to provide more specific delineation of tumor tissue in the brain than ${ }^{18} \mathrm{~F}-\mathrm{FDG}$ (5). Whether the current imaging market is large enough to support the necessary approval process is uncertain, but numerous compounds that are in development for commercial and research purposes could meet the requirements for clinical applications.

However, to our knowledge, no innovative and commercially available candidate compound is currently in development in the radiopharmaceutical industry, although the clinical need has been clearly defined. A major obstacle for the costly tracer development process is the relatively small number of patients with brain tumors. Consequently, and although we already mentioned the need for patentprotected tracers in a highly commercialized market, it seems worthwhile to supplement commercially distributed compounds with "public," already established and proven radiopharmaceuticals, such as ${ }^{18} \mathrm{~F}$-ethyltyrosine.

The limitation of a small imaging market does not apply to the diagnosis of Alzheimer disease, which is prevalent in aging societies. The decision to develop ${ }^{18} \mathrm{~F}$-labeled amyloid ligands has been made. Three tracers are currently in phase III evaluations and may soon be available for widespread clinical applications. The introduction of these tracer compounds is interesting for multiple reasons; first, they do not replace an existing SPECT indication; second, the development of all 3 tracers is backed by large radiopharmaceutical and pharmaceutical companies; and third, this development occurs despite ongoing discussions about whether a specific tracer is needed to diagnose a disease for which no treatment exists.

There are great societal expectations for finding the means to prevent and cure Alzheimer disease. Early diagnosis of the disease process with specific tracers may allow earlier therapy aimed at preventing and slowing the disease process. It will be interesting to observe how national health care agencies react to the approval of new compounds and to what extent screening for early Alzheimer disease is supported by societies.

${ }^{18} \mathrm{~F}-3$,4-dihydroxyphenylalanine is used in many PET centers for the management of suspected or proven Parkinson disease. ${ }^{18} \mathrm{~F}$-labeled markers of neuronal amine transport may be used as substitutes for existing SPECT tracers and may also become useful probes in oncology. Development will be dependent on the clinical need expressed by the neurology community and the willingness of the radiopharmaceutical industry to satisfy this need.

Oncology. Determining how many PET tracers are needed for oncology applications is more difficult. Only a few established SPECT tracers have become key tracers in the oncologic diagnostic process. For many years, ${ }^{131} \mathrm{I}$ and ${ }^{123} \mathrm{I}$ were used effectively for the management of thyroid cancer. ${ }^{124}$ I may provide some advantages (related to subsequent therapy with ${ }^{131}$ I) for a dosimetric estimation based on quantitative PET data. Bone scans have been used for diagnosing skeletal metastases, and an alternative PET procedure is already available. ${ }^{111}$ In-labeled somatostatin analogs have been use for sensitive and specific SPECT identification of neuroendocrine tumors. In the future, ${ }^{68} \mathrm{Ga}$-labeled compounds likely will fully replace ${ }^{111} \mathrm{In}$ tracers at sites with access to PET instrumentation.

There may be a revival of immunoscintigraphy with antibody fragments or minibodies, supported by a new protein format such as Anticalin (Pieris AG). There is a need to develop approaches for adjusting the pharmacokinetics of a compound to the physical properties (half-life) of PET radioisotopes, such as ${ }^{64} \mathrm{Cu}$ and ${ }^{89} \mathrm{Zr}$, to obtain the best possible diagnostic information (6). Because antibody imaging has had limited success in nuclear medicine so far, the results obtained with new PET approaches must be evaluated before clinical need can be addressed.

The role of nuclear medicine in oncology changed dramatically after ${ }^{18}$ F-FDG became commercially available and recognized as a biomarker for malignant tissue. Despite the lack of specificity, the high sensitivity, especially in the context of hybrid instrumentation, helped to rapidly ensure wide clinical acceptance among oncology clinicians and strengthened the role of nuclear medicine worldwide. Nevertheless, more specific tracers with all of the positive characteristics of ${ }^{18}$ F-FDG are needed. Unfortunately, only a few candidates on the horizon are likely to fill the position of the second line of tracers behind ${ }^{18}$ F-FDG. Many new promising PET metabolic tracers are being tested in academia and the radiopharmaceutical industry, but their superiority in the differentiation of malignant and inflammatory tissues has not been satisfactorily documented.

${ }^{18}$ F-FDG has been shown to be of limited value for several oncologic diseases. In tumor entities such as prostate cancer, hepatocellular carcinoma, and gastric cancer, other tumor-specific PET markers are needed to meet clinical requirements. This is especially true for the detection and 
monitoring of prostate cancer, which is one of the most prevalent types of cancer. The clinical success of prostatespecific antigen assays for the early in vitro detection of tumor tissue has increased diagnostic requests for the in vivo localization of primary and recurrent disease. The diagnostic value of other radiologic procedures for this type of cancer does not satisfy clinical requirements, and prostate tissue biopsies are associated with diagnostic uncertainties. This large diagnostic gap has been at least partially addressed by use of PET radiopharmaceuticals such as ${ }^{18} \mathrm{~F}$-labeled cholines, which are sensitive markers for the detection of recurrent or metastatic prostate cancer (7). However, they are of limited value for primary diagnosis because of considerable signal overlap between malignant and nonmalignant pathologies (8). There is no question that sensitive and specific PET tracers for prostate cancer are in demand. Because of the large market for the imaging of prostate cancer, the development of such probes is and will continue to be supported by the radiopharmaceutical industry. Consequently, the evaluation of prostate cancer-specific probes is under way in many academic laboratories and in early clinical trials in the radiopharmaceutical industry.

\section{Generator-Based ${ }^{68}$ Ga PET Tracers}

An important advance in PET tracer development involves the rapidly developing ${ }^{68} \mathrm{Ga}$ chemistry. Various recent reports demonstrated that ${ }^{68} \mathrm{Ga}$ generators can be used efficiently for the labeling of peptides and other imaging compounds. Chelating complexes such as DOTA and NOTA can be used with rapid synthesis methods. Because of its increasing availability, the ${ }^{68} \mathrm{Ge} /{ }^{68} \mathrm{Ga}$ generator system, with a half-life of $270 \mathrm{~d}$, may rival the ${ }^{99 \mathrm{~m}} \mathrm{Tc}$ generator-based radiopharmaceutical system in the future. Local generator-supported radiotracer synthesis may help to transfer most of the existing SPECT radiopharmaceuticals to PET technology and support our first and second hypotheses. The use of ${ }^{68} \mathrm{Ga}$-labeled somatostatin receptor ligands in place of ${ }^{111} \mathrm{In}$-based SPECT radiopharmaceuticals and the introduction of ${ }^{68} \mathrm{Ga}-\mathrm{Galligas}$ (the PET analog of ${ }^{99 \mathrm{~m}} \mathrm{Tc}$-technegas) for pulmonary ventilation in combination with ${ }^{68} \mathrm{Ga}$-macroaggregated alumbins for pulmonary perfusion may make PET methods attractive alternatives to established SPECT methods in many hospitals $(9,10)$. The use of ${ }^{68} \mathrm{Ga}$-citrate for the detection of inflammation may serve as a further example of our second hypothesis regarding the transfer of established imaging markers to PET (11). The clinical acceptance of ${ }^{68} \mathrm{Ga}$ generators as alternatives to ${ }^{99 \mathrm{~m}} \mathrm{Tc}$ generators will depend on the approval of the generator system and the technical details of radiosynthesis under more or less strict good manufacturing practice rules. How many ${ }^{68} \mathrm{Ga}$ tracers are needed? If chemically and technically possible, there should be at least as many ${ }^{68} \mathrm{Ga}$ tracers as

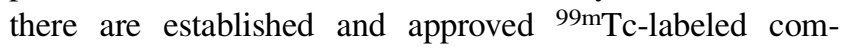
pounds on the market. A variety and an increasing number of ${ }^{68} \mathrm{Ga}$ tracers will expand the pool of alternative PET tracers (12). We are convinced that the availability of approved
${ }^{68} \mathrm{Ga}$ generators will significantly stimulate the development of new ${ }^{68} \mathrm{Ga}$ probes and accelerate corresponding clinical trials. Admittedly, we do not have a realistic perspective toward a ${ }^{68} \mathrm{Ga}$-labeled ${ }^{18} \mathrm{~F}$-FDG analog; however, the frequently used argument against the vision of an on-site radiopharmacy solely based on ${ }^{68} \mathrm{Ga}$ radiopharmaceuticals has never been a limiting factor for ${ }^{99 \mathrm{~m}} \mathrm{Tc}$ and ${ }^{111} \mathrm{In}$ radiopharmaceutical-based SPECT centers; thus, this argument should not impair the future of ${ }^{68} \mathrm{Ga}$, if ${ }^{68} \mathrm{Ga}$ labeling technology is available.

\section{Molecular Imaging}

Molecular imaging is being heralded as the "revolution" of imaging, although the principles may not differ significantly from those of existing conventional nuclear medicine imaging. The concepts for the experimental development of molecular imaging include numerous molecular and cellular targets, which may be of special interest for translation to clinical oncology, cardiology, and neurology. The increasing specificity of a molecular probe for a given pathophysiologic situation or signaling pathways may be advantageous for the prognostic evaluation of a particular subset of patients but may also limit overall diagnostic performance because of the decreasing sensitivity for a given disease in general. The most prominent example of a new molecular imaging probe is a radiopharmaceutical aimed at the amyloid deposits in patients with Alzheimer disease. The development of this tracer is a good example of translational research: amyloid deposition has been described as an integral part of the pathophysiology of Alzheimer disease and, therefore, represents an imaging target related to the molecular basis of the disease process. Obviously, clinical use would be greatly supported by the introduction of specific therapeutic strategies aimed at the same target.

In analogy, several promising PET tracers are currently being used in preclinical and clinical oncologic research. Because many of these tracers target biologic activities, such as proliferation, angiogenesis, and apoptosis, their clinical use most likely will benefit from therapeutic strategies aimed at the same or closely related processes. We believe that these tracers are only of limited use for diagnosis but may be of great value for supporting and strengthening the concept of personalized medicine. Do we need them now? In radiation oncology there is definitely a clinical need to identify hypoxic tissue, which is less sensitive to chemoradiotherapy and chemotherapy. However, the clinical characterization of markers for the quantification of tissue oxygenation, such as ${ }^{18} \mathrm{~F}$-misonidazole, is still inadequate, and further studies must demonstrate the prognostic benefit of biology-based radiation therapy. As a further example, the PET tracer $3^{\prime}$-deoxy- $3^{\prime}-{ }^{18}$ F-fluorothymidine $\left({ }^{18} \mathrm{~F}-\mathrm{FLT}\right)$ has been shown to be specific for the proliferative process of cancer cells. However, compared with ${ }^{18} \mathrm{~F}-\mathrm{FDG}$, ${ }^{18} \mathrm{~F}-\mathrm{FLT}$ has limited sensitivity for the detection of tumor tissue (13). Thus, without clinical evidence that an increased rate of proliferation, as determined with ${ }^{18} \mathrm{~F}-\mathrm{FLT}$, has prog- 
nostic value, clinical acceptance of this tracer may be difficult. On the other hand, this tracer is a typical example of the potential of PET to provide quantitative measurements of specific biologic processes related to oncogenesis as a tool for the in vivo phenotyping of cancer. Initial research results suggest a high sensitivity of ${ }^{18} \mathrm{~F}-\mathrm{FLT}$ imaging for the early detection of a therapy response $(14,15)$. An alteration in the rate of proliferation, as assessed with ${ }^{18} \mathrm{~F}-\mathrm{FLT}$, may help to identify cytostatic responses; in contrast, in most instances, ${ }^{18} \mathrm{~F}-\mathrm{FDG}$ is used to define cytotoxic effects resulting in cell death. Therefore, a major hope for clinical molecular imaging is associated with its use in combination with therapy.

The use of targeted therapies as an integral part of individualized medicine for cancer requires a pretherapeutic assessment of target expression and its possible change as a result of therapy. In addition, imaging procedures for studying the direct effects of drugs on the target, subsequent processes, or correlating pathways are needed. Targets such as Her2 growth receptor expression are already routinely assessed by biopsy and in vitro techniques before the initiation of therapy with the antibody trastuzumab. It is possible that Her2 expression can be quantitatively measured by imaging methods supplementing the pretherapeutic in vitro analysis and allowing the longitudinal monitoring of therapy at multiple tumor sites. The combination of target-specific tracers and dedicated imaging devices in this context needs to be considered. It is likely that the imaging of molecular targets in breast cancer will benefit from multimodal breast imaging technology combining high spatial resolution with biologic specificity.

The early assessment of a therapy response is one of the most important aspects of personalized therapy strategies for cancer. Several experimental protocols in animals and humans have documented the promise of molecular imaging for the early evaluation of a response. ${ }^{18} \mathrm{~F}-\mathrm{FDG}$ PET has been demonstrated to be a valuable tool for the early evaluation of responses in solid and hematologic malignancies (16). We are convinced that after the more formal acceptance of ${ }^{18} \mathrm{~F}-\mathrm{FDG}$ as a surrogate endpoint for response evaluation in accordance with PERCIST criteria (17), other signals will be appreciated to follow the path of integrating size and functional criteria for routine clinical therapy evaluation in cancer patients. It is too early to name tracers that may qualify for this purpose, but the intense research in this area will certainly produce viable candidates. It is anticipated that for each therapeutic target system (such as angiogenesis, tyrosine kinase, growth factors, and oncogenes), representative tracers will be developed to accompany the drug evaluation and validation process and presumably the clinical application as well. Some tracers targeting angiogenic activity have been introduced, but angiogenesis includes numerous and often redundant pathways, limiting the value of only one specific tracer-and one specific drug-as representatives for the entire angiogenesis process. For example, $\alpha_{\mathrm{v}} \beta_{3}$-integrins, expressed on activated endothelial cells, may be an interesting signal for the migration of endothelial cells during angiogenesis. Tracers targeting these proteins may be useful for directing and monitoring therapies with compounds targeting $\alpha_{\mathrm{v}} \beta_{3^{-}}$ integrins, such as Cilengitide (Merck) (18), but may not be suitable for monitoring therapy with vascular endothelial growth factor receptor antagonists. In contrast, less specific and more sensitive metabolic probes may be interesting alternatives for monitoring drug-related biologic effects. Clinical research performed in close cooperation with the pharmaceutical industry is needed to address the value of molecular imaging in the drug development process and may produce new probes that can be translated to the clinic for the routine monitoring of targeted therapy.

Finally, atherosclerotic disease develops over a long time period, and vascular plaque formation and rupture are important pathophysiologic events that are responsible for acute myocardial infarction, stroke, and the development of flowlimiting stenoses in many vascular beds (19). There is no question that a specific biomarker for the early detection of vulnerable plaques is needed. Many research laboratories using different imaging methodologies are pursuing the goal of plaque imaging. Again, the vascular uptake of ${ }^{18} \mathrm{~F}-\mathrm{FDG}$ seems to indicate the invasion of macrophages as a marker of vascular inflammation (20). Further research is required to produce more specific markers that can be used as early signals for atherosclerosis and as surrogate endpoints for therapies aimed at preventing the formation of vulnerable plaques.

\section{CONCLUSION}

On the basis of our hypotheses, a large but definable number of PET tracers is needed to transfer established nuclear medicine imaging procedures to existing and increasing PET/CT-equipped nuclear medicine services. The challenges encountered in establishing such tracers and subsequent reimbursement are expected to be less insurmountable than those associated with new tracers because a large body of clinical experience already exists for the former indications. After the clinical success of ${ }^{82} \mathrm{Rb}$ generators in cardiology, the introduction of a ${ }^{68} \mathrm{Ge} /{ }^{68} \mathrm{Ga}$ generator system together with simple and fast labeling strategies will support and potentially strengthen the development of these tracers. In addition, disease-specific new PET tracers for use in diseases with a high prevalence, such as coronary artery disease, neurodegeneration, infections, and common tumors, such as prostate cancer, will be needed. Ongoing basic and translational research activities in other, common diseases, such as diabetes mellitus ( $\beta$-cell imaging) and psychiatric disorders (e.g., depression), are likely to produce targets that may be exploited for the development of specific tracers. There is no question that this research will be supported and accelerated by the radiopharmaceutical industry.

Numerous probes, such as ${ }^{18} \mathrm{~F}$-ethyltyrosine, ${ }^{18} \mathrm{~F}$-fluoromisonidazole, and ${ }^{18} \mathrm{~F}$-labeled cholines, already have proven clinical efficiency but are not protected by patents. Still to be determined is whether existing suppliers of such tracers 
and upcoming distributors of new tracers, together with professional organizations such as the Society of Nuclear Medicine, the Academy of Molecular Imaging, and the European Association of Nuclear Medicine, can support financially and administratively the process leading to a reimbursed nuclear medicine imaging procedure. The creation of a national registry for ${ }^{18} \mathrm{~F}$ bone scans is a promising example.

Molecular imaging will definitely grow as an important research tool for basic as well as translational research. Competing research programs worldwide will produce many new tracer approaches for single-modality imaging and multimodality imaging, which will serve as an important "pool" of new tracers for clinical applications. The clinical demand for and acceptance of such compounds, however, will depend on the success of existing procedures and new strategies combining imaging and therapy for the realization of personalized medicine.

\section{ACKNOWLEDGMENTS}

The authors thank Dr. Alexander Drzezga for his critical review and Kerstin Wotke for her secretarial support. This work was partially supported by grant SFB 824 from the German Research Society. No other potential conflict of interest relevant to this article was reported.

\section{REFERENCES}

1. Sokoloff L. Mapping local functional activity by measurement of local cerebral glucose utilization in the central nervous system of animals and man. Harvey Lect. 1983;79:77-143.

2. Phelps ME, Mazziotta JC. Positron emission tomography: human brain function and biochemistry. Science. 1985;228:799-809.

3. International Atomic Energy Agency. Directory of cyclotrons used for radionuclide production in member states: 2006 update. Vienna, Austria: International Atomic Energy Agency; 2006. IAEA-DCRP/2006.

4. Nekolla SG, Reder S, Saraste A, et al. Evaluation of the novel myocardial perfusion positron-emission tomography tracer ${ }^{18} \mathrm{~F}-\mathrm{BMS}-747158-02$ : compari- son to ${ }^{13} \mathrm{~N}$-ammonia and validation with microspheres in a pig model. Circulation. 2009;119:2333-2342.

5. Pauleit D, Stoffels G, Bachofner A, et al. Comparison of ${ }^{18} \mathrm{~F}-\mathrm{FET}$ and ${ }^{18} \mathrm{~F}-\mathrm{FDG}$ PET in brain tumors. Nucl Med Biol. 2009;36:779-787.

6. Schlapschy M, Theobald I, Mack H, Schottelius M, Wester HJ, Skerra A. Fusion of a recombinant antibody fragment with a homo-amino-acid polymer: effects on biophysical properties and prolonged plasma half-life. Protein Eng Des Sel. 2007;20:273-284.

7. Picchio M, Briganti A, Fanti S, et al. The role of choline positron emission tomography/computed tomography in the management of patients with prostate-specific antigen progression after radical treatment of prostate cancer. Eur Urol. 2011;59:51-60.

8. Farsad M, Schiavina R, Castellucci P, et al. Detection and localization of prostate cancer: correlation of ${ }^{11} \mathrm{C}$-choline $\mathrm{PET} / \mathrm{CT}$ with histopathologic step-section analysis. J Nucl Med. 2005;46:1642-1649.

9. Maus S, Buchholz HG, Ament S, Brochhausen C, Bausbacher N, Schreckenberger M. Labelling of commercially available human serum albumin kits with ${ }^{68} \mathrm{Ga}$ as surrogates for ${ }^{99 \mathrm{~m}} \mathrm{Tc}-\mathrm{MAA}$ microspheres. Appl Radiat Isot. 2011;69:171-175.

10. Kotzerke J, Andreeff M, Wunderlich G. PET aerosol lung scintigraphy using Galligas. Eur J Nucl Med Mol Imaging. 2010;37:175-177.

11. Nanni C, Errani C, Boriani L, et al. ${ }^{68} \mathrm{Ga}$-citrate PET/CT for evaluating patients with infections of the bone: preliminary results. J Nucl Med. 2010;51:19321936.

12. Fani M, Andre JP, Maecke HR. ${ }^{68}$ Ga-PET: a powerful generator-based alternative to cyclotron-based PET radiopharmaceuticals. Contrast Media Mol Imaging. 2008;3:67-77.

13. Bading JR, Shields AF. Imaging of cell proliferation: status and prospects. J Nucl Med. 2008;49(suppl 2):64S-80S.

14. Graf N, Herrmann K, den Hollander J, et al. Imaging proliferation to monitor early response of lymphoma to cytotoxic treatment. Mol Imaging Biol. 2008; 10:349-355.

15. Herrmann K, Buck AK, Schuster T, et al. Predictive value of initial ${ }^{18}$ F-FLTuptake in patients with aggressive Non-Hodgkin lymphoma receiving R-CHOP treatment. J Nucl Med. 2011;52:690-696.

16. Weber WA. Assessing tumor response to therapy. J Nucl Med. 2009; 50(suppl 1):1S-10S.

17. Wahl RL, Jacene H, Kasamon Y, Lodge MA. From RECIST to PERCIST: evolving considerations for PET response criteria in solid tumors. J Nucl Med. 2009;50(suppl 1):122S-150S.

18. Mas-Moruno C, Rechenmacher F, Kessler H. Cilengitide: the first anti-angiogenic small molecule drug candidate design, synthesis and clinical evaluation. Anticancer Agents Med Chem. 2010;10:753-768.

19. Libby P, DiCarli M, Weissleder R. The vascular biology of atherosclerosis and imaging targets. J Nucl Med. 2010;51(suppl 1):33S-37S.

20. Rudd JH, Hyafil F, Fayad ZA. Inflammation imaging in atherosclerosis. Arterioscler Thromb Vasc Biol. 2009;29:1009-1016. 